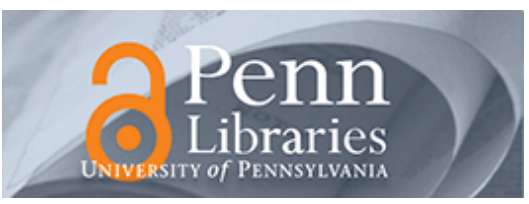

University of Pennsylvania

ScholarlyCommons

April 2007

\title{
Single-Chip Multiple-Frequency ALN MEMS Filters Based on Contour-Mode Piezoelectric Resonators
}

\author{
Gianluca Piazza \\ University of Pennsylvania, piazza@seas.upenn.edu \\ Philip J. Stephanou \\ University of California \\ Albert P. Pisano \\ University of California
}

Follow this and additional works at: https://repository.upenn.edu/ese_papers

\section{Recommended Citation \\ Gianluca Piazza, Philip J. Stephanou, and Albert P. Pisano, "Single-Chip Multiple-Frequency ALN MEMS Filters Based on Contour-Mode Piezoelectric Resonators", . April 2007.}

Copyright 2007 IEEE. Reprinted from Journal of Microelectromechanical Systems, Volume 16, Issue 2, April 2007, pages 319-328.

This material is posted here with permission of the IEEE. Such permission of the IEEE does not in any way imply IEEE endorsement of any of the University of Pennsylvania's products or services. Internal or personal use of this material is permitted. However, permission to reprint/republish this material for advertising or promotional purposes or for creating new collective works for resale or redistribution must be obtained from the IEEE by writing to pubs-permissions@ieee.org. By choosing to view this document, you agree to all provisions of the copyright laws protecting it.

This paper is posted at ScholarlyCommons. https://repository.upenn.edu/ese_papers/238

For more information, please contact repository@pobox.upenn.edu. 


\title{
Single-Chip Multiple-Frequency ALN MEMS Filters Based on Contour-Mode Piezoelectric Resonators
}

\begin{abstract}
This paper reports experimental results on a new class of single-chip multiple-frequency (up to $236 \mathrm{MHz}$ ) filters that are based on low motional resistance contour-mode aluminum nitride piezoelectric micromechanical resonators. Rectangular plates and rings are made out of an aluminum nitride layer sandwiched between a bottom platinum electrode and a top aluminum electrode. For the first time, these devices have been electrically cascaded to yield high performance, low insertion loss (as low as $4 \mathrm{~dB}$ at $93 \mathrm{MHz}$ ), and large rejection ( $27 \mathrm{~dB}$ at $236 \mathrm{MHz}$ ) micromechanical bandpass filters. This novel technology could revolutionize wireless communication systems by allowing cofabrication of multiple frequency filters on the same chip, potentially reducing form factors and manufacturing costs. In addition, these filters require terminations ( $1 \mathrm{kOmega}$ termination is used at $236 \mathrm{MHz}$ ) that can be realized with on-chip inductors and capacitors, enabling their direct interface with standard 50-Omega systems.
\end{abstract}

\section{Keywords}

aluminide nitride (AIN), contour-mode resonators, ladder filters, microelectromechanical systems (MEMS), filters, microelectromechanical systems (MEMS) resonators, piezoelectric resonators

\section{Comments}

Copyright 2007 IEEE. Reprinted from Journal of Microelectromechanical Systems, Volume 16, Issue 2, April 2007, pages 319-328.

This material is posted here with permission of the IEEE. Such permission of the IEEE does not in any way imply IEEE endorsement of any of the University of Pennsylvania's products or services. Internal or personal use of this material is permitted. However, permission to reprint/republish this material for advertising or promotional purposes or for creating new collective works for resale or redistribution must be obtained from the IEEE by writing to pubs-permissions@ieee.org. By choosing to view this document, you agree to all provisions of the copyright laws protecting it. 


\title{
Single-Chip Multiple-Frequency ALN MEMS Filters Based on Contour-Mode Piezoelectric Resonators
}

\author{
Gianluca Piazza, Member, IEEE, Philip J. Stephanou, Member, IEEE, and Albert P. (Al) Pisano
}

\begin{abstract}
This paper reports experimental results on a new class of single-chip multiple-frequency (up to $236 \mathrm{MHz}$ ) filters that are based on low motional resistance contour-mode aluminum nitride piezoelectric micromechanical resonators. Rectangular plates and rings are made out of an aluminum nitride layer sandwiched between a bottom platinum electrode and a top aluminum electrode. For the first time, these devices have been electrically cascaded to yield high performance, low insertion loss (as low as $4 \mathrm{~dB}$ at $93 \mathrm{MHz}$ ), and large rejection ( $27 \mathrm{~dB}$ at $236 \mathrm{MHz}$ ) micromechanical bandpass filters. This novel technology could revolutionize wireless communication systems by allowing cofabrication of multiple frequency filters on the same chip, potentially reducing form factors and manufacturing costs. In addition, these filters require terminations $(1 \mathrm{k} \Omega$ termination is used at $236 \mathrm{MHz})$ that can be realized with on-chip inductors and capacitors, enabling their direct interface with standard $50-\Omega$ systems.

[2006-0051]
\end{abstract}

Index Terms-Aluminum nitride (AIN), contour-mode resonators, ladder filters, microelectromechanical systems (MEMS) filters, microelectromechanical systems (MEMS) resonators, piezoelectric resonators.

\section{INTRODUCTION}

A $S$ THE DEMAND for ubiquitous connectivity grows, the expectations of wireless appliance's functionalities are becoming increasingly exacting. Radio frequency (RF) microelectromechanical systems (MEMS) is an emerging technology that promises to enable both new RF system architectures and unprecedented levels of performance and integration. The principal drivers of research in RF-MEMS resonator technology are resonator-based circuits, namely filters and oscillators. Solutions capable of integrating multiband and multistandard devices that consume low power and have small form factors will accomplish the vision of next-generation, ubiquitous wireless communications.

Several research groups [1]-[7] have demonstrated coupled electrostatically driven microresonators for filtering applications. Although characterized by very high $\mathrm{Q}$, these microdevices suffer from large motional resistance, $\mathrm{R}_{\mathrm{M}}$. For

Manuscript received March 23, 2006; revised October 28, 2006. This work was supported by DARPA by Grant No. NBCH1020005. Subject Editor S. Lucyszyn.

G. Piazza is with the Department of Electrical and Systems Engineering, University of Pennsylvania, Philadelphia, PA 19104 USA (e-mail: piazza@seas. upenn.edu).

P. J. Stephanou and A. P. Pisano are with the Department of Mechanical Engineering, University of California, Berkeley, CA 94720 USA (e-mail: stephp@newton.berkeley.edu; appisano@me.berkeley.edu).

Color versions of one or more of the figures in this paper are available online at http://ieeexplore.ieee.org.

Digital Object Identifier 10.1109/JMEMS.2006.889503 filters, a large $R_{M}$ translates either into the need for bulky matching elements or large insertion losses and makes these resonators unintegrable with existing $50-\Omega$ systems. Recent research results [8]-[12] have shown the ability to lower the motional resistance of these microresonators by using "internal" electrostatic transduction. Although promising, this technology remains unproven and must contend with large capacitances. Piezoelectric materials such as aluminum nitride or quartz offer larger electromechanical coupling coefficients that reduce the motional resistance of the resonators to a few ohms and provide large bandwidths for filtering applications. Piezoelectric resonators, such as film bulk acoustic resonators (FBARs) [13]-[19] have been successfully demonstrated and electrically cascaded to form bandpass filters in the GHz range. Because the film thicknesses determine the center frequency of these resonators, multiple-frequency filtering solutions based on FBARs cannot be economically manufactured as single-chip RF modules.

For the first time, this paper realizes monolithic integration of multiple-frequency aluminum nitride (AlN) bandpass filters, which represents a major breakthrough towards the goal of highly integrated, single-chip, multiband solutions. Using a new class of MEMS resonator technology based on the excitation of contour modes of vibration in AlN microstructures [17], bandpass filters at 93 and $236 \mathrm{MHz}$ have been demonstrated by electrically cascading up to eight resonators in a ladder topology. These filters show very promising performance, being characterized by low insertion losses ( $4 \mathrm{~dB}$ at $93 \mathrm{MHz})$, large close-in and out-of-band rejection $(\sim 40$ and $\sim 27 \mathrm{~dB}$, respectively, for a $93-\mathrm{MHz}$ filter) and fairly sharp roll-off with a $20 \mathrm{~dB}$ shape factor of 2.2. The filters described in this paper are about $20 \times$ smaller than existing surface acoustic wave (SAW) device based implementations. In addition, the $-25 \mathrm{ppm} /{ }^{\circ} \mathrm{C}$ temperature coefficient of contour mode AlN filters results in $40 \%$ lower temperature sensitivity than some of their (e.g., $\mathrm{LiTaO}_{3}$ ) SAW counterparts.

\section{RESONATOR ARRAYS}

Vast amounts of research in the field of electromechanical resonators for filtering applications has generated different techniques for realizing bandpass filters. The same fundamental methods that have undergone refinement and innovation for over 60 years [20]-[22] are still the preferred implementions for bandpass filters using resonators. There are two main approaches:

1) electrically coupled filters, in which an array of resonators is coupled together exclusively by electrical signals; the resonators are cascaded in series and parallel branches to 

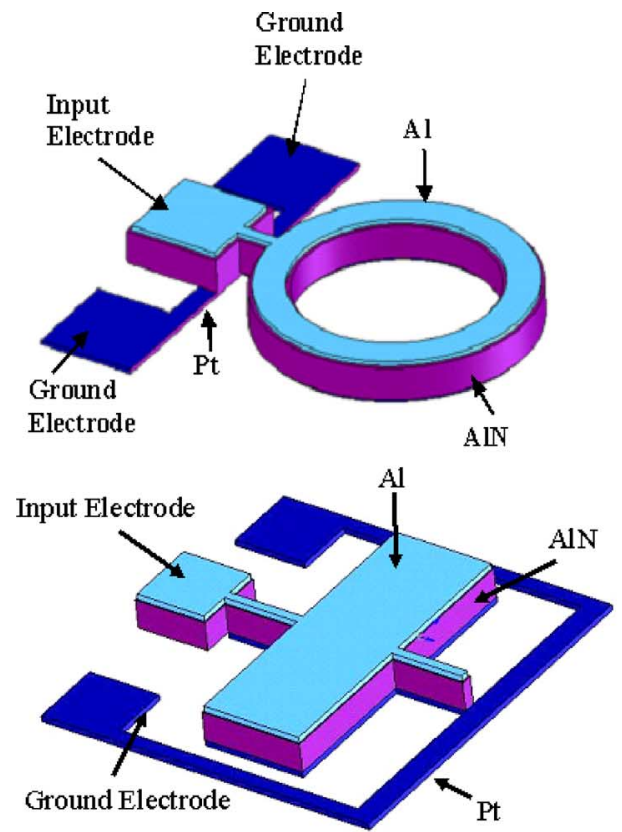

(a)

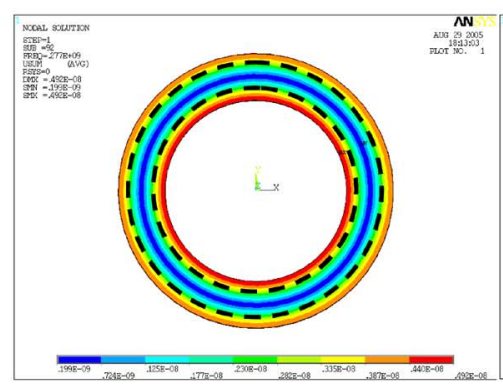

(b)

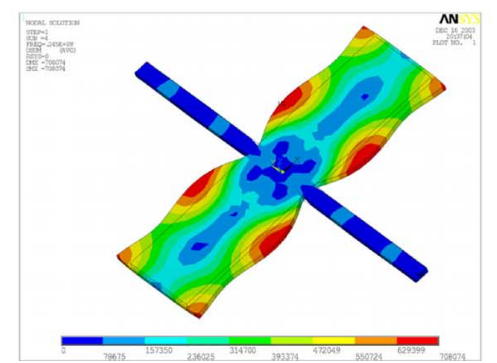

Fig. 1. Schematic views of the building blocks for the filters and their mode shapes. (a) Circular ring AlN resonator excited in a radial-extensional contour mode shape as shown in ANSYS plot. (b) Rectangular plate AIN resonator excited in a width-extensional contour mode shape as shown in ANSYS plot.

form either by themselves or with external passive components (inductors and capacitors) L, PI, or T networks;

2) mechanically coupled filters, in which arrays of resonators are coupled by purely mechanical links; different arrangements of resonators are possible so that fully mechanical signal processing functions can be implemented. The electrical signal is simply used to excite the input of the structure and is picked off at the output stage, whereas the bandpass function is constructed by purely mechanical interactions between the resonators.

Between the two approaches, electrical coupling of resonators allows the advantage of simpler implementation. There is no need for external mechanical links, which are generally very challenging to manufacture especially at high frequencies. The size of the link is generally a fraction of the resonator size $(\sim 1 / 10)$ and at $1 \mathrm{GHz}$ could translate into critical dimensions below $0.5 \mu \mathrm{m}$. In addition, electrical cascading of resonators offer rapid implementation of complex designs with zeros (also known as attenuation poles) in the filter transfer function. Advantages of mechanically coupled resonators include the ability to set the bandwidth of the filter independently of material properties [23] and the ability to improve out-of-band rejection. Theoretically the use of mechanically coupled resonators eliminates the limit on the maximum achievable bandwidth imposed by the material properties, and increases the out-of-band rejection by eliminating the parallel capacitance of the device. Thus a hybrid solution that combines mechanical and electrical coupling of resonators might be very intriguing, especially for contour mode resonators. In this paper, because of their ease of design and fabrication, electrically coupled resonators are presented as an example of the implementation of IF filters. The following sections provide details on the design and experimental characterization of these filters.

\section{DESIGN OF EleCtRICALly COUPLED FILTERS}

The AlN resonators are formed by a thin $(2 \mu \mathrm{m})$ AlN film sandwiched between a bottom platinum (Pt) electrode and a top aluminum (Al) electrode. By applying an electric field across the film thickness, the resonator is excited in lateral vibrations by means of the $d_{31}$ piezoelectric coefficient. The contour-mode rectangular plate and ring-shaped AIN resonators shown in Fig. 1 are the building blocks for the bandpass filters of this paper. The basic ladder filter configuration is composed of cascaded L networks (Fig. 3), each formed by series and shunt resonators. Such networks can be cascaded to form PI and $\mathrm{T}$ networks and other more complicated multipole filters.

In order to analyze these networks, a concise way of describing the equivalent electrical impedance $Z_{R}$ of each oneport resonator is adopted [based on the Butterworth Van Dyke (BVD) model] [24]

$$
\begin{aligned}
z_{R} & =\frac{1}{j \omega C_{o}} \frac{z_{S}}{z_{P}} \\
z_{S} & =1-\left(\frac{f}{f_{S}}\right)^{2}+j\left(\frac{f}{f_{S}}\right) / Q_{S} \\
z_{P} & =1-\left(\frac{f}{f_{P}}\right)^{2}+j\left(\frac{f}{f_{P}}\right) / Q_{P}
\end{aligned}
$$

where $f_{S}$ and $f_{P}$ are the series and parallel resonant frequencies, $Q_{S}$ and $Q_{P}$ are the resonator's quality factor for the series and parallel resonances, and $C_{o}$, is the parallel capacitance of the BVD model shown in Fig. 2. $C_{o}$ is used instead of an equivalent low frequency capacitance as reported in [24] because the two only differ by approximately $1 \%$. 


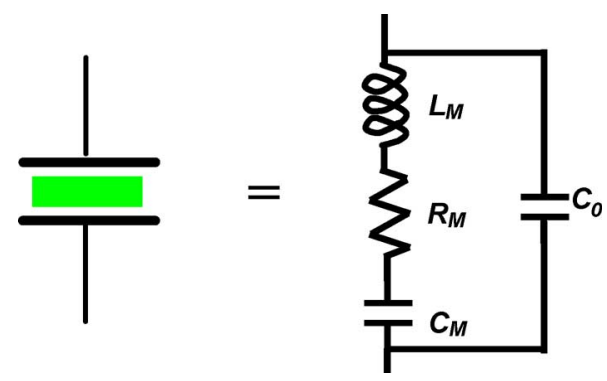

Fig. 2. Schematic representation of a piezoelectric resonator and equivalent BVD circuit representation.

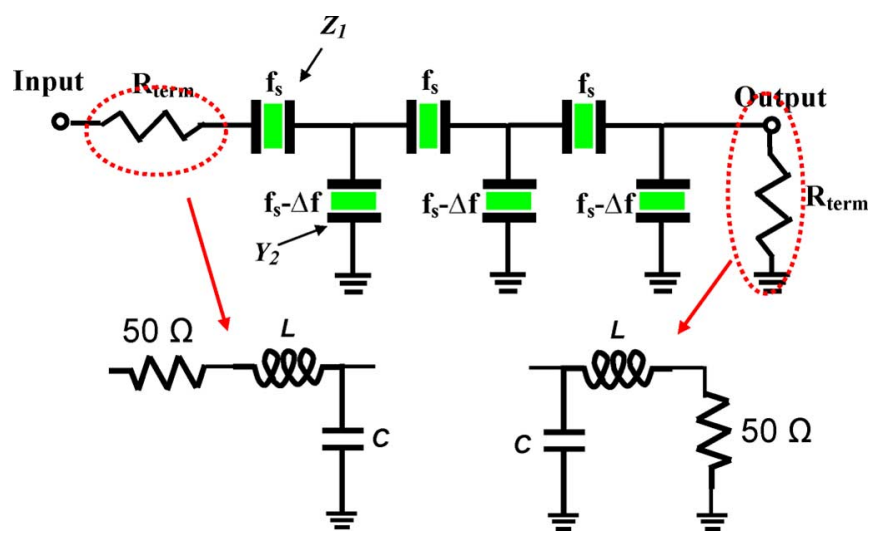

Fig. 3. Schematic representation of a ladder filter structure made out of three cascaded $\mathrm{L}$ networks. $L C$ matching elements can be used to implement the termination resistance and directly couple the system match to $50 \Omega$.

Adopting the ABCD matrix approach [25], each L section can be described in the following way:

$$
\left[\begin{array}{ll}
A & B \\
C & D
\end{array}\right]=\left[\begin{array}{cc}
1+Y_{2} Z_{1} & Z_{1} \\
Y_{2} & 1
\end{array}\right]
$$

where

$$
Z_{1}=\frac{1}{j \omega C_{1}} \frac{z_{S 1}}{z_{P 1}} \quad Y_{2}=j \omega C_{2} \frac{z_{P 2}}{z_{S 2}}
$$

and the subscript 1 refers to the series branch resonator and 2 to the shunt branch resonator. $C_{1}$ and $C_{2}$ represent the resonator parallel capacitance $C_{o}$, respectively, for the series branch and the shunt branch resonators. Therefore, using the correspondence between $\mathrm{ABCD}$ matrix elements and scattering parameters [25] it is possible to derive the scattering parameter of an L section. Specifically, $S_{21}$ is given by (4), shown at the bottom of the page, where $R_{\text {term }}$ is the termination resistance. By simply cascading one section after the other one can construct more complex filter structures

Equation (4) shows a maximum when the series resonance of the series branch, $f_{S 1}$, coincides with the parallel resonance of the shunt branch, $f_{P 2}$. In order to correctly match the filter characteristic impedance at its center frequency, the termination resistance $R_{\text {term }}$ should be chosen to be [26]

$$
R_{\mathrm{term}} \approx \frac{1}{\omega \sqrt{C_{1} C_{2}}} \approx R_{M} \frac{Q_{\mathrm{RES}}}{Q_{\mathrm{FLTR}}}=\frac{M_{\mathrm{RES}}}{\eta_{\mathrm{RES}}^{2}} B W_{\mathrm{FLTR}}
$$

where $Q_{\text {RES }}$ and $Q_{\text {FLTR }}$ are the quality factor of the single resonator and of the filter ( $3 \mathrm{~dB}$ bandwidth of filter), respectively, $M_{\mathrm{RES}}$ is the equivalent mass of the resonator, $\eta_{\mathrm{RES}}$ is the electromechanical coupling factor for the resonator [27], specifically $\eta_{\mathrm{RES}}=2 E_{P} d_{31} L$ for a rectangular plate resonator, $E_{P}$ being the AlN Young's moduls, $d_{31}$ is the piezoelectric coefficient, and $L$ is the length of the plate, and $B W_{\text {FLTR }}$ is the $3 \mathrm{~dB}$ filter bandwidth. It is clear from (5) that a large $R_{M}$ requires multiple matching stages that introduce loss. At the same time, high Q cannot compensate for small electromechanical coupling (typical of electrostatically driven resonators) to reduce $R_{\text {term }}$ for a given filter bandwidth.

For a given value of $R_{\text {term }}$, the insertion losses $I L$ of the filter can be expressed in the following way:

$$
I L \approx 1-\frac{n}{4 \pi f_{C} C_{1} R_{\text {term }} k_{t}^{2} Q_{\mathrm{RES}}}
$$

where $k_{t}^{2}$ is the effective electromechanical coupling coefficient and $n$ refers to the number of series resonators that are cascaded to form the filter. $k_{t}^{2}$, which describes the internal conversion between electrical and mechanical energy, is a material dependent property that does not depend on the specific geometry of the transducer under consideration ( $k_{\mathrm{t}}^{2}$ is different from $\left.\eta_{\mathrm{RES}}\right)$

$$
k_{t}^{2}=\frac{d_{i j}^{2}}{\varepsilon_{i} s_{j j}}
$$

where $d_{i j}$ is the specific piezoelectric coefficient in use ( $d_{31}$ for contour-mode resonators), $\varepsilon_{i}$ is the dielectric permittivity of the piezoelectric material, and $s_{j j}$ is its compliance. Because of its generality, $k_{t}^{2}$ is considered an important figure of merit in estimating the performance of a resonator for filtering applications. $k_{t}^{2}$ does not only affect the $I L$ of the filter, but also sets its fractional bandwidth (9). Out-of-band rejection of a ladder filter can be computed from (4) when the frequency is far from the filter's

$$
\begin{aligned}
S_{21} & =\frac{2}{A+D+B / R_{\mathrm{term}}+C R_{\mathrm{term}}} \\
S_{21} & =\frac{2 j \omega C_{1} z_{P 1} z_{S 2}}{2 j \omega C_{1} z_{P 1} z_{S 2}+j \omega C_{2} z_{S 1} z_{P 2}+z_{S 1} z_{S 2} / R_{\mathrm{term}}-\omega^{2} C_{2} C_{1} z_{P 2} z_{P 1} R_{\mathrm{term}}}
\end{aligned}
$$




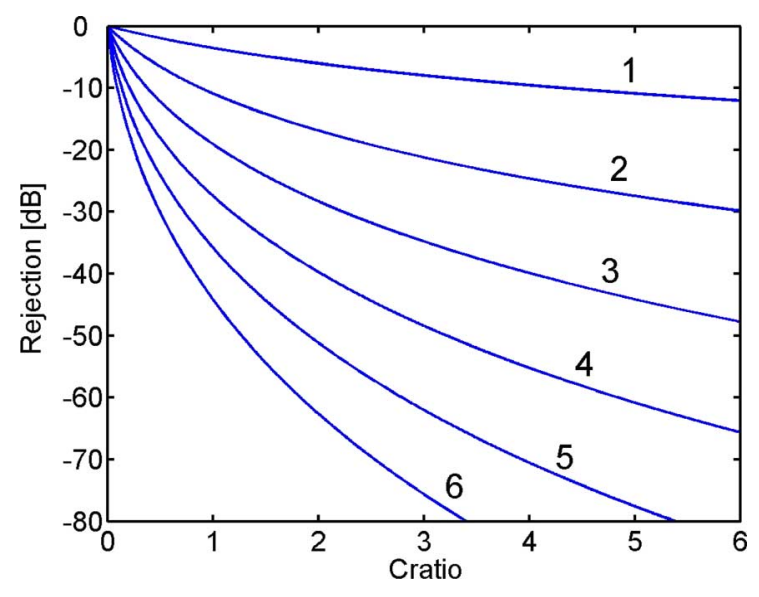

Fig. 4. Effect of capacitance ratio (between parallel and series branches) and number of $\mathrm{L}$ sections (here varying between 1 and 6) on the out-of-band rejection.

center frequency. The out-of-band value of $S_{21}$ for $n$ L stages is approximately

$$
S_{21 \_ \text {Out-of-Band }} \approx \frac{1}{\left(1+\frac{C_{2}}{C_{1}}\right)^{n}}
$$

and depends primarily on the capacitance ratio of the resonators in the shunt and series branches. The dependence of the out of band rejection on the capacitor ratio and the number of $\mathrm{L}$ sections is shown in Fig. 4 [exact calculation based on (4) was used in Fig. 4].

A particular feature of the ladder filter transfer function is the presence of two zeros, coinciding, respectively, with the series resonance of the shunt resonator $f_{S 2}$ and the parallel resonance of the series resonator, $f_{P 1}$. These zeros provide for sharp roll-off of the filter and high close-in-band rejection. This feature is particularly important when designing duplexers, which demand large isolation between closely spaced transmitting and receiving channels. These zeros also set the fractional bandwidth of the filter according to

$$
\frac{f_{P 1}-f_{S 2}}{f_{C}} \approx \frac{8}{\pi^{2}} k_{t}^{2} .
$$

Based on (9), the maximum achievable fractional bandwidth for contour-mode resonators is about $2.5 \%$. Therefore, unless a hybrid approach that uses both mechanically and electrically coupled resonators is adopted, it is problematic to obtain the larger fractional bandwidths (up to $3 \%$ ) that cellular standards such as PCS, GSM, and WCDMA require for RF filters. The behavior of the L section of a ladder filter can be described in an intuitive manner as depicted in Fig. 5. Far from resonance (region A and E), the two resonators behave as capacitor, and the transfer function is given by the ratio of the two capacitors. At the series resonance (region B) of the shunt resonator, $f_{S 2}$, the shunt resonator behaves as a very small resistance to ground, yielding a zero in the transfer function. Similarly at the parallel resonance (region $\mathrm{D}$ ) of the series resonator, $f_{P 1}$, the series resonator can be approximated by an open (very large resistance) and again
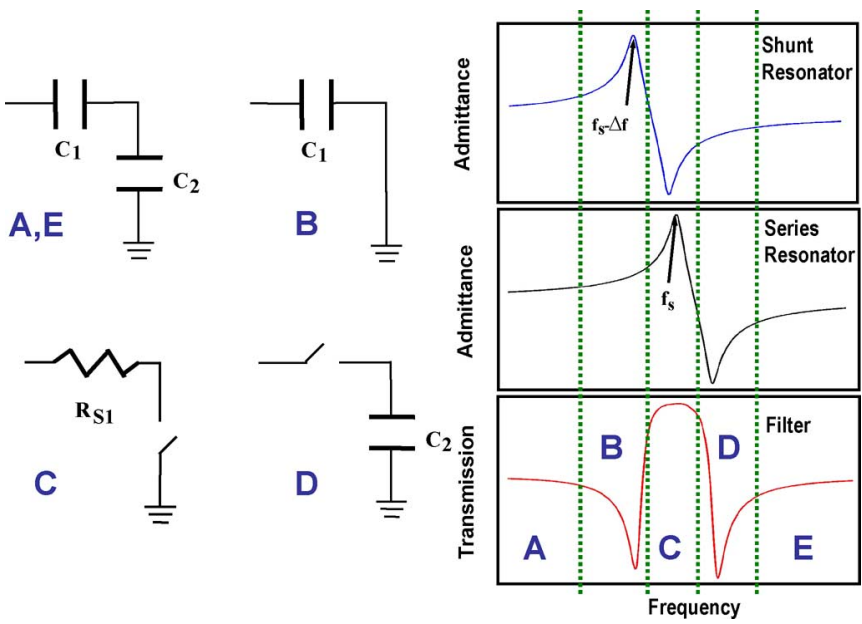

Fig. 5. Intuitive explanation of the principle of operation of the $\mathrm{L}$ section used as a building block in ladder filters. Five principle areas of operation have been identified and the corresponding equivalent circuit of each of the resonators has been highlighted.

a zero appears in the filter transfer function. In the pass band (region C) the series resonators show a minimum resistance responsible for the insertion losses, whereas the shunt branches behave as open circuits.

According to the aforementioned derivations, the designer has a set of parameters with which to prescribe the performance of a ladder filter:

1) the center frequency by properly selecting the desired contour geometry;

2) the capacitance ratio between the series and parallel branches. This choice affects not only the out-of-band rejection, but also the insertion losses, termination resistance, geometry and motional resistance. In addition, in some solutions [28], instead of including different size resonators, it is advisable to employ two resonators of the same size coupled in series or parallel in order to reduce or increase the effective capacitance;

3) the number of stages and the number of resonators in the series and parallel branches. The number of stages directly affects the order of the filter and therefore its roll-off and shape factor. The number of series and parallel resonators to be employed affects the overall size of the filter and changes the actual shape of the transfer function (Fig. 6). Several solutions are possible from this point of view and are generally dictated by a tradeoff between space savings and satisfying the specifications of the relevant standards.

\section{FREQUENCY SHIFT}

An important feature for the implementation of ladder filters is the ability to manufacture resonators with different resonant frequencies for the series and shunt branches; such difference needs to be comparable to the $3 \mathrm{db}$ bandwidth of the filter. As outlined in the previous section, it is of fundamental importance to have the series resonance of the series resonator overlapping with the parallel resonance of the shunt resonator. Depending on the bandwidth specification, this may require a frequency shift in the range of $0.1 \%$ and $3 \%$. 

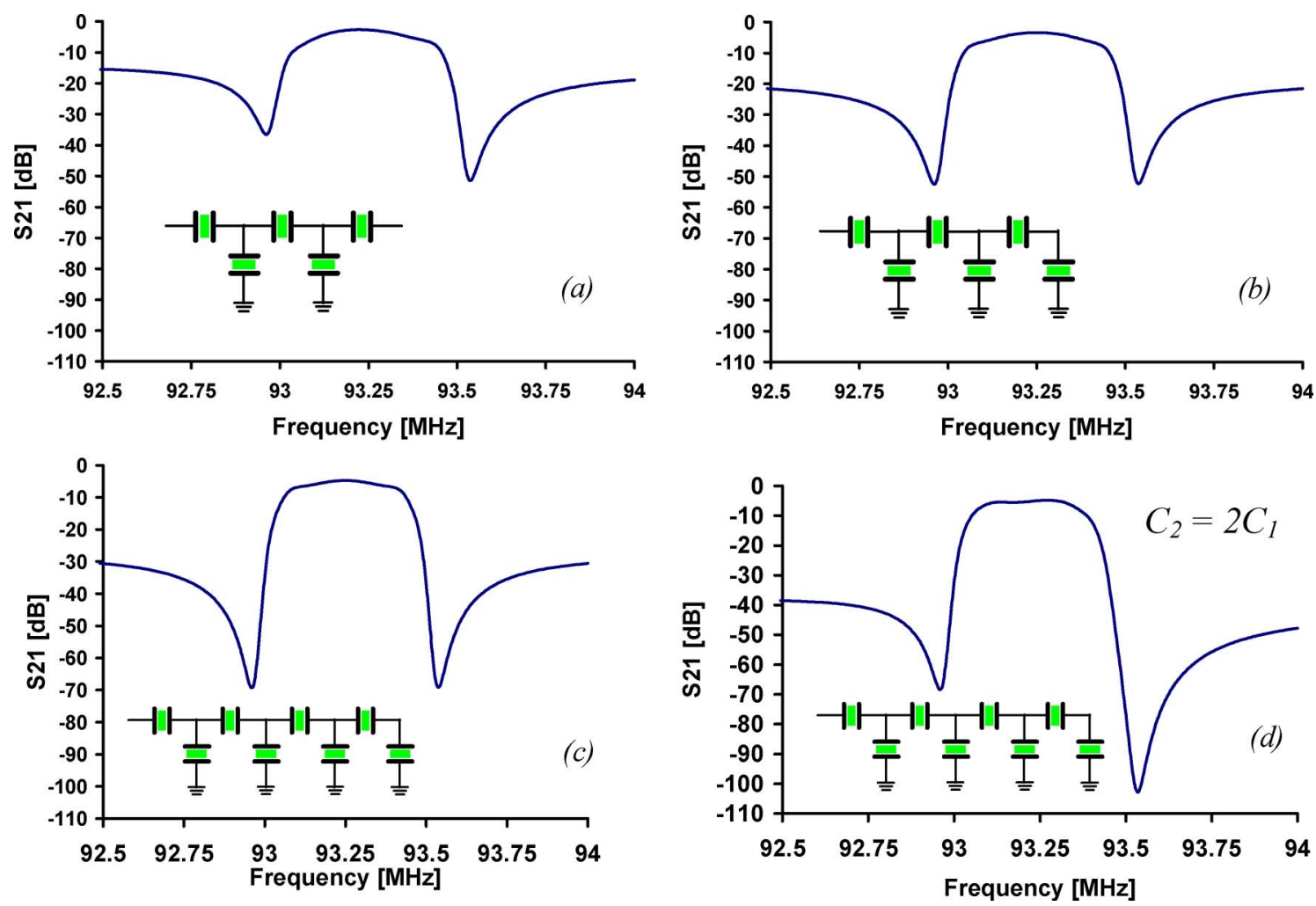

Fig. 6. Simulated $S_{21}$ response of a 93-MHz filter implemented using rectangular plate resonators having individual $\mathrm{Q}$ of 2000 and $\mathrm{R}_{\mathrm{M}}$ of $300 \Omega$. The termination resistors were selected according to equation (5). (a) Electrical response of 3/2 ladder structure showing asymmetry and poor out-of-band rejection. (b) Electrical response of 3/3 ladder structure showing higher rejection and symmetrical response. (c) Electrical response of 4/4 ladder structure; higher out-of-band rejection is obtained, but insertion losses are increased with respect to (b). (d) Electrical response of $4 / 4$ ladder structure for which a cap ratio of 2 between the parallel and series branches was selected [ $C_{1}$ and $C_{2}$ as defined in (3)]. This configuration improves out-of-band rejection with a small increase in insertion loss.

Although the frequency of contour-mode resonators is determined by the in-plane geometrical dimensions of the structure, it is not practical to define such a small shift by simply changing the dimensions of the structure at the CAD level. This change would in fact require prohibitively expensive submicron accuracy on the lithography equipment. The preferred solution involves exploiting the mass loading caused by the Pt electrode. Pt has a mass density which is about 6.5 times that of AlN. By lithographically removing small amounts of $\mathrm{Pt}$ it is possible to raise the center frequency of the resonator. This is a significant feature of the present paper, because it allows the desired frequency shift to be implemented at the mask layout level, thereby obviating the need for additional deposition steps as is generally required for thickness mode resonators. This solution drastically simplifies the fabrication process making it more robust and economical. It is worth noting from Fig. 7 (cross-sectional view of a ring) that the removal of Pt occurs at the location of minimum strain. According to the piezoelectric equations [29], we can remove small amounts of Pt without affecting the electrical transfer function of the resonator because minimal motional charge is generated at these locations.

The analysis of the frequency shift induced by Pt is presented below. Selectively removing $\mathrm{Pt}$ in a pattern that is perpendicular to the direction of bulk acoustic wave propagation in the piezoelectric medium allows us to use a very simple modeling approach based on [30]. This approach proves valid when removing a small amount of Pt from the length of a rectangular

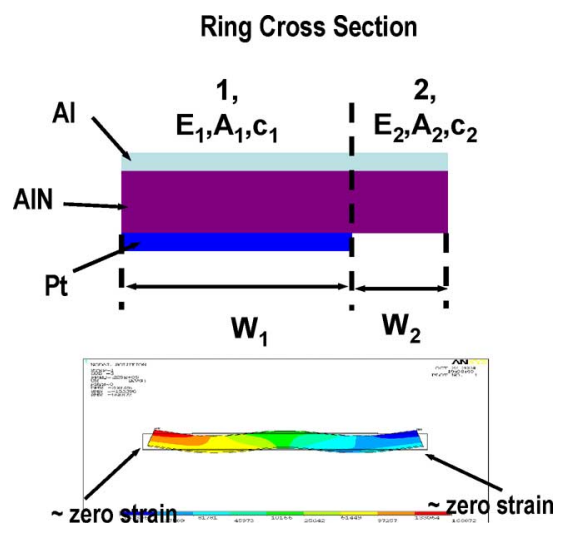

Fig. 7. Cross-sectional view of circular ring structure illustrating the location where $\mathrm{Pt}$ is lithographically removed in order to induce the desired frequency shift. Note that the Pt is removed at the location of minimum or almost zero strain as shown by cross-sectional view of resonator mode shape in ANSYS.

plate excited in a width-extensional mode. In this case we can find that the frequency of vibration is given by [30]

$$
f_{\mathrm{NEW}}=f_{o} \sqrt{\frac{\left(1+\frac{E_{P t} A_{P t}}{E_{R} A_{R}}\right)}{\left(1+\frac{\rho_{P t} A_{P t}}{\rho_{R} A_{R}}\right)}}
$$

where $f_{o}$ is the resonator center frequency without any metal; $E, A$, and $\rho$ are the equivalent Young's modulus, cross-sectional 
area and mass density of either platinum (subscript Pt) or the piezoelectric material (subscript $R$ ). By changing the length of the Pt electrode, the effective area of the Pt layer $A_{P t}$ changes and according to (10) the resonator frequency can be raised. It is possible to induce a frequency shift of $500 \mathrm{ppm}$ for every $\mu \mathrm{m}$ of Pt that is removed from the length of a $200 \times 50 \mu \mathrm{m}$ rectangular plate having a $100-\mathrm{nm}$-thick Pt electrode.

The case of the ring, as shown in Fig. 7, is different because the removal of $\mathrm{Pt}$ occurs in the same direction of propagation of the bulk wave. The problem needs to be handled as a two-part composite rod undergoing longitudinal vibrations [31]. In this case we need to define an equivalent " $E A$ " (product of Young's modulus time the cross-sectional area) and an equivalent acoustic velocity for sections 1 and 2 in Fig. 7. Therefore, (Al electrode is considered negligible in this analysis)

$$
\begin{aligned}
E_{1} A_{1} & =E_{P t} A_{P t}+E_{P} A_{P} \quad E_{2} A_{2}=E_{P} A_{P} \\
A_{P t} & =T_{P t} 2 \pi R_{A V E} \quad A_{P}=T_{P} 2 \pi R_{A V E} \\
c_{1} & =\sqrt{\frac{E_{1} A_{1}}{\rho_{P t} A_{P t}+\rho_{P} A_{P}}} \quad c_{2}=\sqrt{\frac{E_{2} A_{2}}{\rho_{P} A_{P}}}=\sqrt{\frac{E_{P}}{A_{P}}}
\end{aligned}
$$

where each area $A$ is an approximate value based on a circular ring geometry, valid for rings with $R_{A V E} \gg W$ and $T_{P t}$ is the thickness of the Pt electrode.

The frequency equation is given by [31]

$$
\begin{aligned}
\frac{E_{2} A_{2}}{E_{1} A_{1}} \cos \left(\frac{\omega}{c_{1}} W_{1}\right) \sin & \left(\frac{\omega}{c_{2}} W_{2}\right)+ \\
& \cos \left(\frac{\omega}{c_{2}} W_{2}\right) \sin \left(\frac{\omega}{c_{1}} W_{1}\right)=0
\end{aligned}
$$

where $\omega$ is the unknown resonant frequency and $W_{1}$ and $W_{2}$ are the width of each section, respectively. According to (12), when small amounts of Pt are removed, variations in the resonator center frequency with respect to $W_{2}$ can be approximated to have a linear dependence on $W_{2}$. Calculations show that for a $100-n m$-thick Pt electrode the center frequency of a $90-\mu \mathrm{m}$ inner radius ring structure shifts by $\sim 7000 \mathrm{ppm}$ for each $\mu \mathrm{m}$ of $\mathrm{Pt}$ that is removed from the width of the structure.

A second method to obtain the desired frequency shift is to use in the series and parallel branches rings with different average diameters or rectangular plates with different aspect ratios. It can be shown (Fig. 8) [32] that the resonant frequency of circular rings is a weak function of the average radius. A decrease in resonant frequency of $0.7 \%$ is recorded when $R_{A V E}$ is increased by three times (from 50 to $150 \mu \mathrm{m}$ ). At the same time, this approach permits one to design different capacitance ratios between the series and shunt resonators, improving the out-of-band rejection, as previously shown in Fig. 6. In contrast to the approach based on Pt etching, this method requires relatively large layout area to define significant (1\%-2\%) frequency shifts and reduces the designer's freedom of setting bandwidth independently of capacitance ratio.

\section{Filter FABRICATION}

The process employed for fabricating the resonant microstructures of this paper is based on surface micromachining techniques that are compatible with state-of-the-art CMOS production systems. The metallization steps are conventional

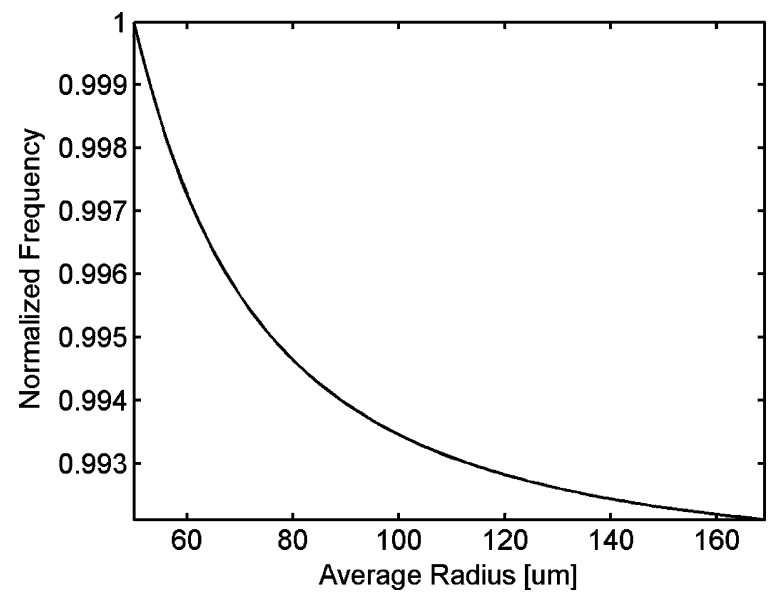

Fig. 8. Frequency variation versus average radius of a $20-\mu \mathrm{m}$-wide circular ring. Frequency is normalized to the value for a ring with $50-\mu \mathrm{m}$ average radius. Rings with larger average radius have lower resonant frequency.

micromachining processes. Although compatible with CMOS fabrication, the etching of AlN and the release of the microstructures can be considered somewhat exotic. In the last decade etching of some III-V compounds such as AlN and $\mathrm{XeF}_{2}$ release are gaining wider use and understanding, but they are still considered unproven for high yield and large volume manufacturing. Despite these issues, the fabrication process of these filters is very robust and very easily implemented.

A simple four-mask process has been used to fabricate the filters (Fig. 9). Two $\mu \mathrm{m}$ AlN film is sandwiched between a bottom platinum electrode and a top aluminum electrode. The fabrication process starts by patterning the bottom Pt electrode (approximately $100 \mathrm{~nm}$ ) on a low stress nitride (LSN) buffer layer in order to reduce parasitic capacitance and electrical losses. The Pt electrode is deposited by RF sputtering and patterned by lift off. The patterning of the Pt electrode improves not only the electrical isolation to the substrate, but also the quality (degree of orientation) of the AlN films. AlN films are sputter-deposited using a single-module Advanced Modular Sputtering (AMS, Goleta, CA) PVD sputtering tool. A wet etch step in $160{ }^{\circ} \mathrm{C}$ phosphoric acid is used to access the bottom electrode. The wet etch step provides for low-slope profiles so that contact between thin Al layers (150-175 nm thick) and Pt bottom electrode is easily achieved. Fig. 9 shows a side and front view of the contact. Unfortunately the deposition of $400{ }^{\circ} \mathrm{C}$ low-temperature oxide (LTO) masking layer for the AlN etch seems to affect the surface roughness of the contact. It is believed that a platinum aluminide alloy forms when the two contacting materials are exposed to high temperatures (see phase diagram of $\mathrm{Pt}$ and $\mathrm{Al}$ in [33]). Despite the roughness, the electrical quality of the contact is preserved. The top $\mathrm{Al}$ electrode is patterned by dry etching. $\mathrm{Cl}_{2}$-based dry etching is used to pattern AlN. The top $\mathrm{Al}$ electrode is protected by a thin (approximately $30 \mathrm{~nm}$ ) layer of $\mathrm{Nb}$ that is removed with the remaining oxide during a $\mathrm{CF}_{4}$-based dry etch step. This step selectively removes the masking layer and etches the remaining LSN without attacking the resonant structure. The selectivity between LTO and AlN during the $\mathrm{Cl}_{2}$-based dry etch process is about 1:3. The structures are released by dry etching of silicon in $\mathrm{XeF}_{2}$, which 


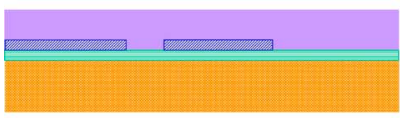

(a)

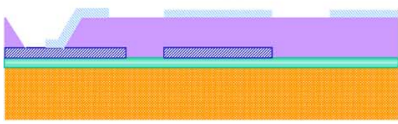

(c)

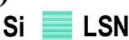

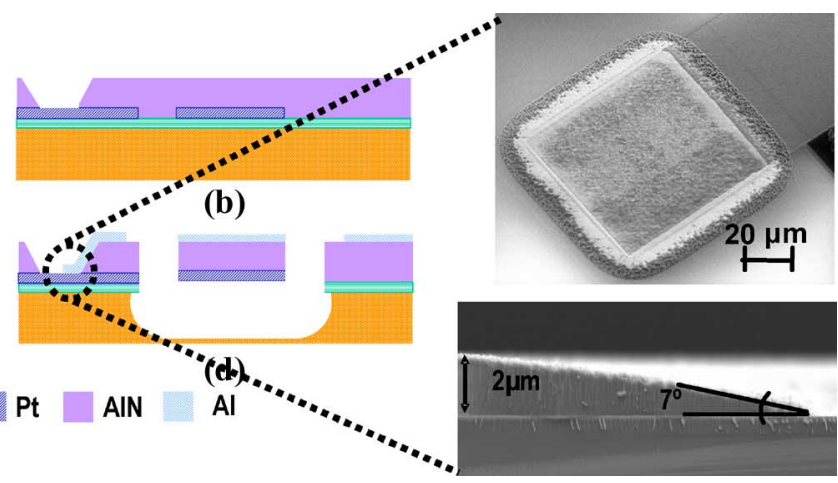

Fig. 9. Fabrication process used for the realization of ladder filters. (a) Low stress nitride (LSN) deposition by LPCVD, followed by Pt patterning by lift-off and AlN sputter deposition. (b) Open via access to bottom Pt electrode through AlN. AlN is wet etched by $160{ }^{\circ} \mathrm{C} \mathrm{H}_{3} \mathrm{PO}_{4}$. (c) Deposition of top Al electrode and patterning by dry etching in $\mathrm{Cl}_{2}$-based chemistry. (d) $\mathrm{Cl}_{2}$-based dry etching of AlN resonant devices and dry release in $\mathrm{XeF}_{2}$. The inset $\mathrm{SEMs}$ show top and side views of the $\mathrm{Al}$ to Pt contact.

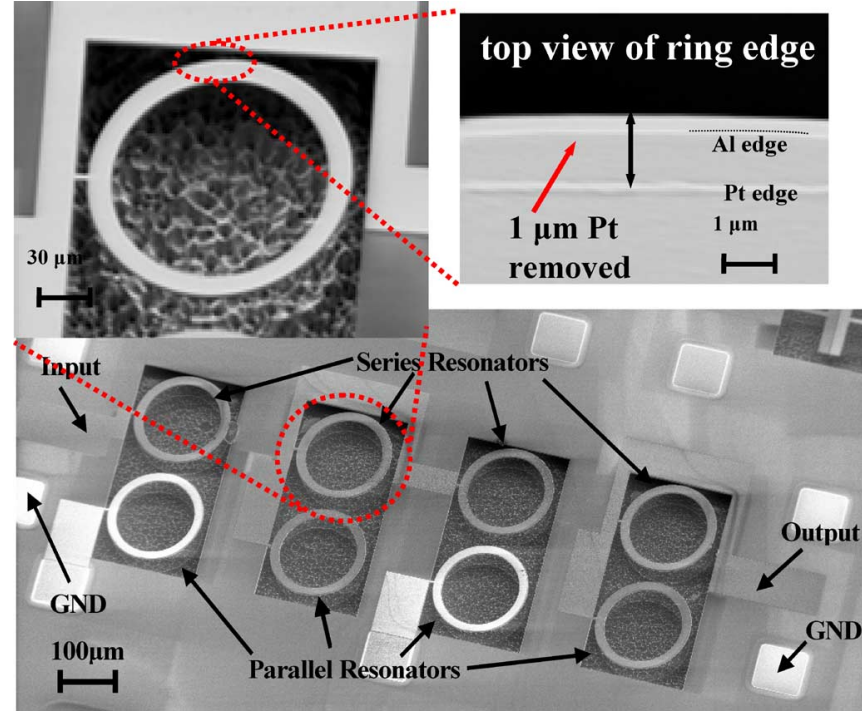

Fig. 10. SEM image of a fourth-order ladder filter made out of eight identical circular rings. The process-defined frequency shift defined by the removal of $1 \mu \mathrm{m}$ of Pt from the series resonator is highlighted in the close-up views.

is extremely selective to $\mathrm{AlN}, \mathrm{Al}$, and $\mathrm{Pt}$, and solely removes $\mathrm{Si}$ and LSN (generally low-Q material) present underneath the structure.

\section{EXPERIMENTAL RESULTS}

The fabricated micromechanical filters were tested in an RF probe station at atmospheric pressure. Ground-signal-ground (GSG) probes were used. Two-port S-parameter calibration based on short, open, load, and through (SOLT) standards on a Picoprobe (GGB Industries, Inc., Naples, FL) ceramic substrate was performed. Full S-parameter matrices were extracted for each filter using an Agilent E5071B network analyzer (Agilent Technologies, Santa Clara, CA). No external terminations were connected to the device under test. The network analyzer allows automatic change of the filter terminations and computation of the transmission spectrum.

Eight rings (Fig. 10), all with an inner radius of $90 \mu \mathrm{m}$ and $20 \mu \mathrm{m}$ wide, were electrically cascaded in a ladder structure. The frequency of the series and shunt branches were shifted by approximately $0.3 \%$. It is important to note that a wider bandwidth could be obtained, but extra parasitic capacitance introduced by the fabrication process and the test fixture limited the overall bandwidth. This filter shows moderate insertion losses of $-7.9 \mathrm{~dB}$ at $236.2 \mathrm{MHz}$, an out-of-band rejection of $26 \mathrm{~dB}$ and a $20 \mathrm{~dB}$ shape factor of 2.79 (Fig. 11). It is interesting to note that this filter does not suffer from any other spurious resonance (Fig. 11). The non-ideal shape factor is due to slight mismatches in frequency between adjacent resonators. The agreement between experimental and theoretical results (obtained by cascading ABCD parameters in (2) is not perfect because parasitic capacitances were not modeled and it was not possible to measure the quality factor and motional resistance of each resonator and eventual mismatch between resonators. It is important to note that a termination of only $1 \mathrm{k} \Omega$ was required for this filter. This value translates into $\mathrm{L}$ and $\mathrm{C}$ components (Fig. 3) of $147 \mathrm{nH}$ and $2.9 \mathrm{pF}$, respectively, values that can be realized by on-chip inductors and capacitors. The combination of on-chip matching to $50 \Omega$ and relatively low insertion losses are the key features of these IF filters. The most important filter performance are also summarized in Table I, where experimental results are compared to theoretical calculations obtained using (4)-(6) and (8). There is good agreement between theory and experiments.

As discussed previously, the frequency shift was also defined by changing the size of the inner radius of the rings in the shunt branch (average radius of $150 \mu \mathrm{m}$ was used in the shunt branch and $100 \mu \mathrm{m}$ in the series branch). Up to six rings were connected in this configuration. Although the results are promising (Fig. 12), large insertion losses ( 11.3 dB) at $236 \mathrm{MHz}$ were recorded, which were attributable to the longer traces needed to connect the resonators. Also, the additional capacitance due to the longer traces affects the out-of-band rejection, negating the effect of an improved capacitance ratio.

Four, six and eight (Fig. 13) 200- $\mu \mathrm{m}$-long and 50- $\mu \mathrm{m}$-wide rectangular plates were tested in a ladder configuration as well. Again the frequencies were shifted by about $0.3 \%$. Although this frequency shift is not sufficient to satisfy the standards at $\mathrm{RF}$ frequencies, it works well for IF frequencies. An example of the electrical response of eight rectangular resonators is shown in Fig. 14. In this case insertion losses as low as $4 \mathrm{~dB}$ were 


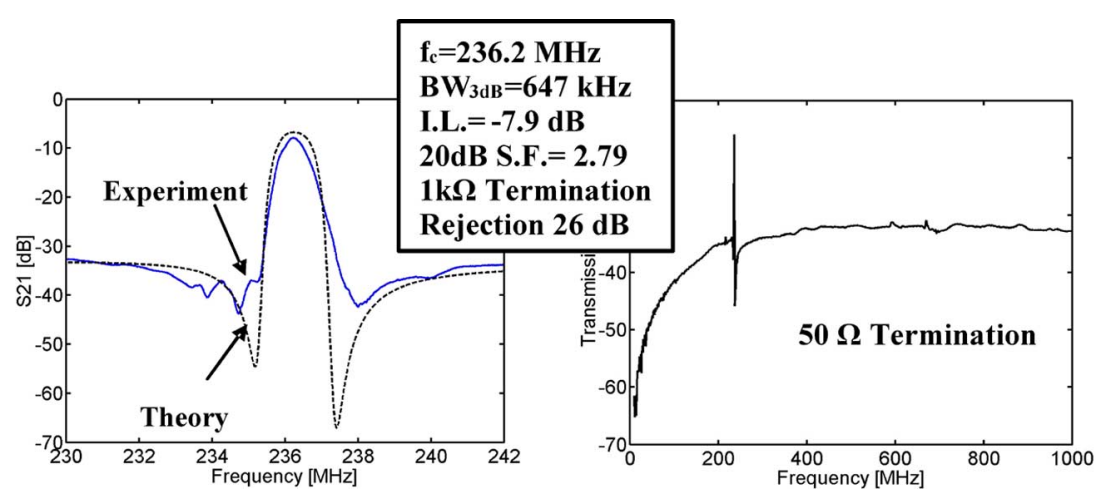

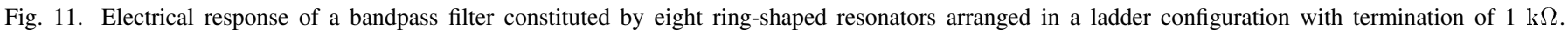

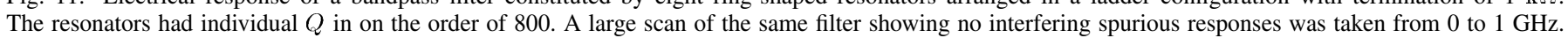
$50-\Omega$ terminations were used in this scan (1-GHz-wide scan).

TABLE I

COMPARISON BETWEEN THEORY AND EXPERIMENT OF THE PRIMARY Performance Parameters For the 8 Ring and 8 Rectangular Plate Filters That Were PRESENTEd IN THIS PAPER AND SHOWN IN Figs. 10 AND 13, RESPECTIVELY. THEORETICAL CALCULATIONS ARE BASED ON THE EXPERIMENTALLY DERIVED RESONATOR PARAMETERS. FOR THE CIRCULAR RING: $C_{o}=0.56 \mathrm{pF}, k_{t}^{2}=0.43 \%, Q=800$. FOR THE RECTANGULAR Plate: $C_{o}=0.81 \mathrm{pF}, k_{t}^{2}=0.4 \%, Q=2000$

\begin{tabular}{|l|c|c|c|c|}
\hline \multirow{2}{*}{$\begin{array}{l}\text { Filter } \\
\text { Performance }\end{array}$} & \multicolumn{2}{|c|}{ 8 Circular Ring Filter } & \multicolumn{2}{c|}{ 8 Rectangular Plate Filter } \\
\cline { 2 - 5 } & Theory & Experiment & Theory & Experiment \\
\hline IL [dB] & -7.3 & -7.9 & -2.5 & -4 \\
\hline Rejection $[\mathbf{d B}]$ & -20 & -26 & -25 & -27 \\
\hline Termination $[\mathbf{\Omega}]$ & 1200 & 1000 & 2110 & 2000 \\
\hline
\end{tabular}
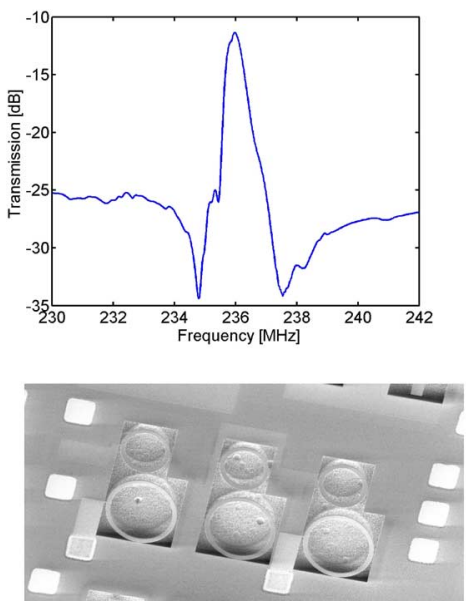

$3+3$ rings
Different size ser/shunt
$\mathrm{f}_{\mathrm{c}}=\mathbf{2 3 6} \mathrm{MHz}$
$\mathrm{BW} \mathrm{HB}_{3 \mathrm{~d}}=53 \mathrm{kHz}$
I.L. $=-11.3 \mathrm{~dB}$
$20 \mathrm{~dB}$ S.F. $=4.4$
$1 \mathrm{k} \Omega$ Termination
Rejection $15 \mathrm{~dB}$

Fig. 12. Electrical response and SEM of a 236-MHz ladder filter realized using different size rings in the series and shunt branches.

recorded at $93.2 \mathrm{MHz}$ and an out-of-band rejection of $27 \mathrm{~dB}$ were achieved. As shown in Fig. 14 a second bandpass filter exists due to the length-extensional mode shape present in the plate. This mode could be suppressed by placing the anchors of the plate along the length instead of the width of the structure [34]. For this filter, a $2-k \Omega$ termination was needed. The value is slightly larger than what was recorded for the filters made out of rings and it is because the rectangular plates show slightly larger motional resistance than the rings. Again on-chip inductors and capacitors could be used for matching, with $\mathrm{L}$ equal to $530 \mathrm{nH}$ and $\mathrm{C}$ to $5.3 \mathrm{pF}$. Table I compares theory and experiments for the filter composed of eight rectangular plates. Discrepancies in

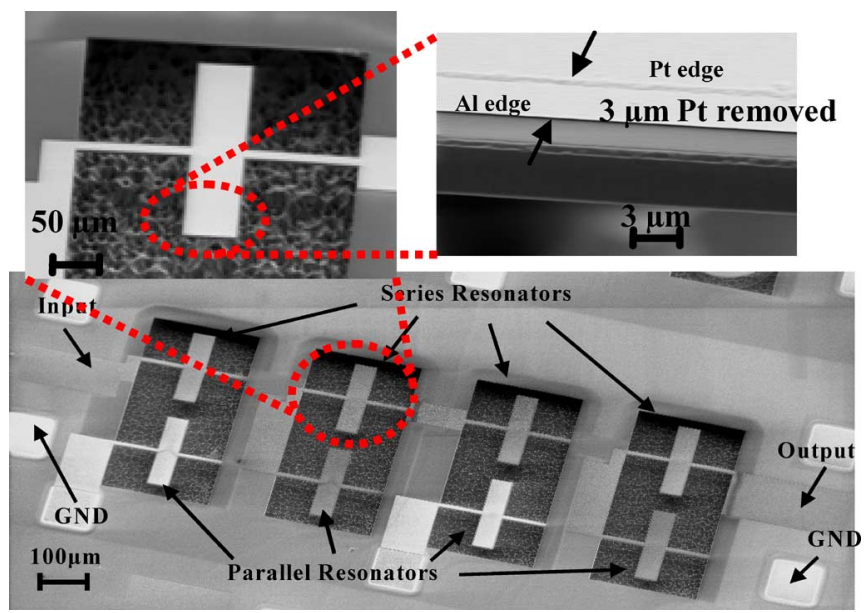

Fig. 13. SEM view of a ladder filter made out of eight electrically cascaded rectangular plate resonators. Zoomed-in view shows details of the single rectangular resonator and lithographically defined variation in bottom electrode length used to tune the center frequency of the series resonators.

the out-of-band rejection are potentially introduced by parasitic capacitances in the shunt branches as for the filter formed by eight rings. The larger than predicted IL are due to performance differences between the different resonators in the filter.

The results for four and six rectangular resonant filters are summarized in Table II. From Table II and Fig. 15 it is evident that a higher-order filter (more L sections) improves the out-ofband rejection at the expenses of larger insertion losses.

These IF filters also showed temperature dependence that is about $40 \%$ better than some existing SAW filters. The TCF of each individual resonator is about $-25 \mathrm{ppm} /{ }^{\circ} \mathrm{C}$. Fig. 16 shows the shift in bandwidth with temperature for a third-order filter that uses six rectangular plates connected in a ladder structure.

\section{CONCLUSION}

For the first time, contour-mode AlN piezoelectric resonators have been electrically coupled in a ladder structure to form low insertion loss, bandpass filters at 93 and $236 \mathrm{MHz}$. Although the performance of these filters are not yet optimized, they represent a major breakthrough toward the realization of single-chip, multifrequency, and integrated bandpass filter solutions characterized by higher performance and smaller form factors than 


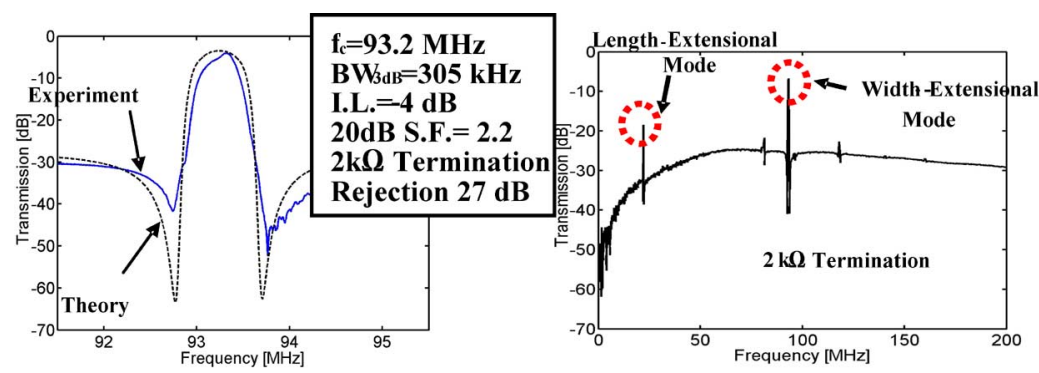

Fig. 14. Electrical response of a 93.2-MHz bandpass filter constituted by eight rectangular resonators arranged in a ladder configuration. Resonators had individual $\mathrm{Q}$ in the order of 2000. Large scan of the same filter response from 0 to $200 \mathrm{MHz}$ with $2-\mathrm{k} \Omega$ terminations was taken. A length-extensional mode is present together with the width-extensional mode.

TABLE II

RESPONSE PARAMETERS AND CHARACTERISTICS OF BANDPASS FILTERS Built Using Rectangular Plate Resonators

\begin{tabular}{|c|c|c|c|c|c|}
\hline $\begin{array}{c}\text { Number of } \\
\text { Resonators }\end{array}$ & $\begin{array}{c}\mathbf{f}_{\mathbf{c}} \\
{[\mathbf{M H z}]}\end{array}$ & $\begin{array}{c}\mathbf{B W}_{\mathbf{3 d B}} \\
{[\mathbf{k H z}]}\end{array}$ & $\begin{array}{c}\mathbf{I . L} . \\
{[\mathbf{d B}]}\end{array}$ & $\begin{array}{c}\mathbf{2 0 d B} \\
\mathbf{S . F} .\end{array}$ & $\begin{array}{c}\mathbf{R}_{\text {term }} \\
{[\mathbf{k} \boldsymbol{\Omega}]}\end{array}$ \\
\hline 4 & 93.5 & 456 & -2 & N.A. & 1.5 \\
\hline 6 & 93.1 & 332 & -4.7 & 2.7 & 2 \\
\hline
\end{tabular}

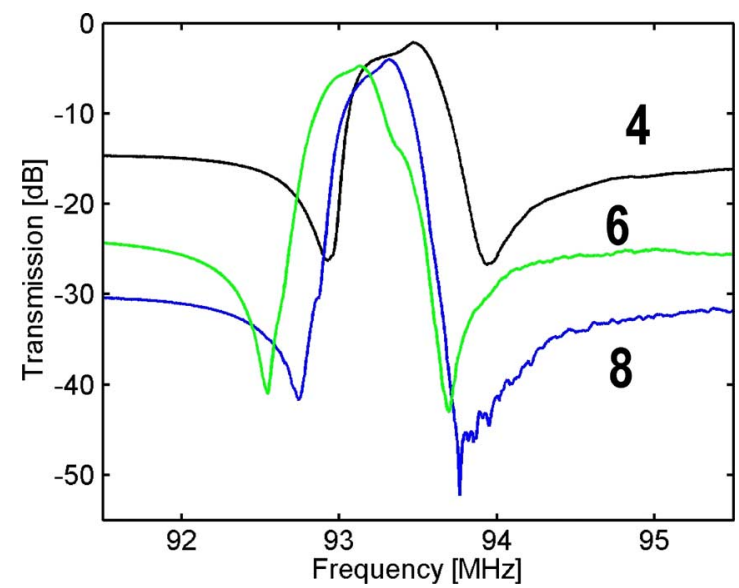

Fig. 15. Electrical response of second-, third-, and fourth-order ladder filters made out of, respectively 4,6 , and 8 rectangular plate resonators. Out-of-band rejection improves with filter order at the expenses of insertion losses.

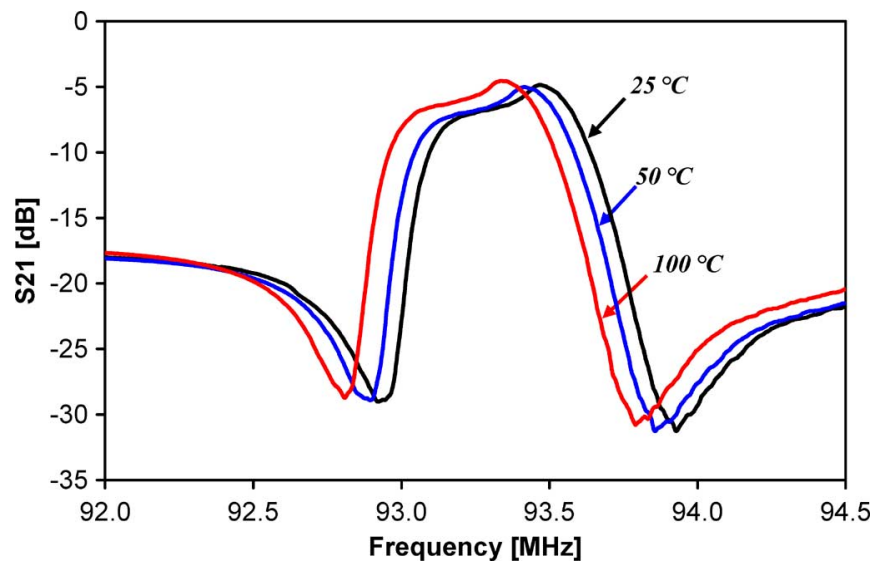

Fig. 16. Effect of temperature change $\left(25-100^{\circ} \mathrm{C}\right)$ on ladder filter composed of six rectangular plate resonators.

existing SAW and quartz crystal filters. Ongoing research is focusing on expanding the bandwidth of the filters, as well as introducing this technology into the $\mathrm{GHz}$ frequencies.

\section{ACKNOWLEDGMENT}

The authors wish to thank the Microfabrication Laboratory staff at the University of California at Berkeley for their help.

\section{REFERENCES}

[1] F. D. Bannon, J. R. Clark, and C.-C. Nguyen, "High-Q HF microelectromechanical filters," IEEE J. Solid-State Circuits, vol. 35, pp. 512-526, 2000.

[2] W. Kun and C.-C. Nguyen, "High-order medium frequency micromechanical electronic filters," J. Microelectromech. Syst., vol. 8, pp. 534-556, 1999.

[3] W. Kun, F. D. Bannon, III, J. R. Clark, and C.-C. Nguyen, "Q-enhancement of microelectromechanical filters via low-velocity spring coupling," in Proc. Ultrason. Symp. , 1997, vol. 1, pp. 323-327.

[4] S. A. Bhave, G. Di, R. Maboudian, and R. T. Howe, "Fully-differential poly-SiC Lame mode resonator and checkerboard filter," in Proc. Int. Conf. Micro Electro Mech. Syst., (MEMS 2005), 2005, pp. 223-226.

[5] G. K. Ho, R. Abdolvand, and F. Ayazi, "Through-support-coupled micromechanical filter array," in Proc. Int. Conf. Micro Electro Mech. Syst., (MEMS 2004), 2004, pp. 769-772.

[6] S. Pourkamali, R. Abdolvand, G. K. Ho, and F. Ayazi, "Electrostatically coupled micromechanical beam filters," in Proc. Int. Conf. Micro Electro Mech. Syst., MEMS 2004, 2004, pp. 584-587.

[7] W. Ark-Chew and C.-C. Nguyen, "Micromechanical mixer-filters ("mixlers")," J. Microelectromech. Syst., vol. 13, pp. 100-112, 2004.

[8] H. Chandrahalim, D. Weinstein, and S. A. Bhave, "Thickness shear mode vibrations in silicon bar resonators," in Proc. Ultrason. Symp., 2005, pp. 898-901.

[9] S. A. Bhave and R. T. Howe, "Internal electrostatic transduction for bulk MEMS resonators," in Proc. 2004 Solid State Sens., Actuator and Microsyst. Workshop, 2004, pp. 59-60.

[10] _ " "Silicon nitride-on-silicon bar resonator using internal electrostatic transduction," in Proc. 13th Int. Conf. Solid-State Sens., Actuators and Microsyst. Transducers '05, 2005, pp. 2139-2142.

[11] H. Chandrahalim, D. Weinstein, L. F. Cheow, and S. A. Bhave, "Channel-select micromechanical filters using high-K dielectrically transduced MEMS resonators," in Proc. Int. Conf. Micro Electro Mech. Syst. (MEMS 2006), 2006, pp. 894-897.

[12] L. Yu-Wei, L. Sheng-Shian, X. Yuan, R. Zeying, and C. T. C. Nguyen, "Vibrating micromechanical resonators with solid dielectric capacitive transducer gaps," in Proc. Freq. Control Symp. Expo., 2005, pp. $128-134$.

[13] K. M. Lakin, J. Belsick, J. F. McDonald, and K. T. McCarron, "Improved bulk wave resonator coupling coefficient for wide bandwidth filters," in Proc. Ultrason. Symp. , 2001, vol. 1, pp. 827-831.

[14] R. Ruby, P. Bradley, D. Clark, D. Feld, T. Jamneala, and W. Kun, "Acoustic FBAR for filters, duplexers and front end modules," in Proc. MTT-S Int. Microw. Symp. Dig., 2004, vol. 2, pp. 931-934.

[15] W. Kun, M. Frank, P. Bradley, R. Ruby, W. Mueller, A. Barfknecht, and M. Gat, "FBAR Rx filters for handset front-end modules with waferlevel packaging," in Proc. Ultrason. Symp., 2003, vol. 1, pp. 162-165.

[16] K. Wang, W. Mueller, R. Ruby, M. Gat, P. Bradley, A. Barfknecht, F. Geefay, C. Han, G. Gan, A. Chien, and B. Ly, "High rejection Rx filters for GSM handsets with wafer level packaging," in Proc. Ultrason. Symp., 2002, vol. 1, pp. 925-929. 
[17] P. Bradley, R. Ruby, J. D. Larson, III, Y. Oshmyansky, and D. Figueredo, "A film bulk acoustic resonator (FBAR) duplexer for USPCS handset applications," in Proc. MTT-S Int. Microw. Symp. Dig., 2001, vol. 1, pp. 367-370.

[18] K. M. Lakin, "Bulk acoustic wave coupled resonator filters," in Proc. Int. Freq. Control Symp. PDA Exhib., 2002, pp. 8-14.

[19] K. M. Lakin, J. R. Belsick, J. P. McDonald, K. T. McCarron, and C. W. Andrus, "Bulk acoustic wave resonators and filters for applications above 2 GHz," in Proc. MTT-S Int. Microw. Symp. Dig., 2002, vol. 3, pp. 1487-1490.

[20] K. M. Lakin, "Coupled resonator filters," in Proc. Ultrason. Symp. , 2002, vol. 1, pp. 901-908.

[21] W. G. Cady, Piezoelectricity; an Introduction to the Theory and Applications of Electromechanical Phenomena in Crystals. New York: McGraw-Hill, 1946.

[22] R. A. Johnson, Mechanical Filters in Electronics. New York: Wiley, 1983.

[23] P. J. Stephanou, G. Piazza, C. D. White, M. B. J. Wijesundara, and A. P. Pisano, "Mechanically coupled contour mode piezoelectric aluminum nitride MEMS filters," in Proc. Int. Conf. Micro Electro Mech. Syst., MEMS, 2006, pp. 906-909.

[24] K. M. Lakin, G. R. Kline, and K. T. McCarron, "Thin film bulk acoustic wave filters for GPS," in Proc. Ultrason. Symp. , 1992, vol. 1, pp. $471-476$.

[25] D. M. Pozar, Microwave Engineering, 3rd ed. New York: Wiley, 2005.

[26] A. I. Zverev, Handbook of Filter Synthesis. New York: Wiley, 1967.

[27] G. Piazza and A. P. Pisano, "Dry-released post-CMOS compatible contour-mode aluminum nitride micromechanical resonators for VHF applications," in Proc. Solid-State Sensor, Actuator and Microsyst. Workshop, Hilton Head, SC, 2004, pp. 37-40.

[28] H. Byeoungju, S. Insang, P. Yunkwon, K. Duckhwan, K. Woonbae, N. Kuangwoo, and P. J. Jungho, "Novel 1-chip FBAR filter for wireless handsets," in Solid-State Sensors, Actuators and Microsyst., Transducers '05, 2005, pp. 2069-2073.

[29] T. Ikeda, Fundamentals of Piezoelectricity. Oxford, U.K.: Oxford Univ. Press, 1990.

[30] W. Weaver, Jr., S. P. Timoshenko, and D. H. Young, Vibration Problems in Engineering. New York: Wiley, 1990.

[31] K. F. Graff, Wave Motion in Elastic Solids. New York: Dover, 1991.

[32] G. Ambati, J. F. W. Bell, and J. C. K. Sharp, "In-plane vibrations of annular rings," J. Sound Vibrat., vol. 47, pp. 415-432, 1976.

[33] L. F. Mondolfo, Mettallography of Aluminum Alloys. New York: Wiley, 1943.

[34] G. Piazza, "Piezoelectric aluminum nitride vibrating RF MEMS for radio front-end technology," Ph.D. dissertation, Univ. Calif., Berkeley, 2005.
Gianluca Piazza (S'00-M'06) received the Ph.D. degree from the University of California at Berkeley, in 2005.

$\mathrm{He}$ is an Assistant Professor with the Department of Electrical and Systems Engineering (ESE), University of Pennsylvania. His research interests focus on piezoelectric micro and nano systems (MEMS/NEMS) for RF wireless communications, bio/chemical detection, wireless sensor platforms, and medical ultrasounds. He also has general interest in the areas of micro/nano fabrication techniques and integration of micro/nano devices with state-of-the-art electronics. $\mathrm{He}$ developed a new class of AlN contour-mode vibrating microstructures for RF communications at the University of California, Berkeley. He has more than five years of experience working with piezoelectric materials, holds two patents in the field of micromechanical resonators and cofounded a start-up company (Harmonic Devices, Inc.) for the commercialization of piezoelectric resonators.

Philip J. Stephanou (M'05) received the B.S. and M.S. degrees from Rensselaer Polytechnic Institute, Troy, NY, in 2001 and 2002, respectively. His thesis investigated the performance of novel algorithms for computational multirigidbody dynamics.

$\mathrm{He}$ is pursuing the Ph.D. degree with the Mechanical Engineering Department, University of California at Berkeley, since 2002. His current research involves the analysis, design and fabrication of piezoelectric RF-MEMS resonators and filters for highly integrated communication systems. In spring 2005, he cofounded Harmonic Devices, Inc. (HDI), a fabless semiconductor startup committed to commercializing research breakthroughs in the area of RF-MEMS for the mobile handset market. He is currently the acting CTO of HDI.

Albert ("Al") P. Pisano received the B.S., M.S., and Ph.D. degrees from Columbia University, New York, NY, in mechanical engineering.

He currently serves as Professor and Chair of the Department of Mechanical Engineering, University of California at Berkeley (UCB). At UCB, he holds the FANUC Chair of Mechanical Systems in the Department of Mechanical Engineering, with a joint appointment to the Department of Electrical Engineering and Computer Science. He currently serves as a Director of the Berkeley Sensor and Actuator Center (BSAC). Prior to joining the faculty at UCB, he held research positions with Xerox Palo Alto Research Center, Singer Sewing Machines Corporate R\&D Center, and General Motors Research Labs. From 1997 to 1999 , he served as Program Manager for the MEMS program at the Defense Advanced Research Projects Agency (DARPA). His research interests and activities at UCB include MEMS for a wide variety of applications, including RF components, power generation, drug delivery, strain sensors, biosensors, and disk-drive actuators. He is the co-inventor listed on 20 patents in MEMS and has authored or coauthored more than 190 archival publications. He is a cofounder in start-up companies in the area of transdermal drug delivery, transvascular drug delivery, sensorized catheters, MEMS manufacturing equipment, and MEMS RF devices.

Dr. Pisano was elected to the National Academy of Engineering in 2001. 